\title{
Inhibition of 5-lipoxygenase in hepatic stellate cells alleviates liver fibrosis
}

Shiyun $\mathrm{Pu}^{1,2 \#}$, Yanping Li ${ }^{2 \#}$, Qinhui Liu ${ }^{2}, \mathrm{Xu}_{\mathrm{Zhang}}{ }^{3}$, Lei Chen ${ }^{1,2}$, Rui Li ${ }^{1,2}$, Jinhang Zhang ${ }^{1,2}$, Tong $\mathrm{Wu}^{1,2}$, Qin Tang ${ }^{1,2}$, Xuping Yang ${ }^{1,2}$, Zijing Zhang ${ }^{1,2}$, Ya Huang ${ }^{1,2}$, Jiangying Kuang ${ }^{1,2}$, Hong $\mathrm{Li}^{1,2}$, Min Zou ${ }^{1}$, Wei Jiang ${ }^{4}$, and Jinhan $\mathrm{He}^{1,2 *}$

${ }^{1}$ Department of Pharmacy and State Key Laboratory of Biotherapy, West China Hospital, Sichuan University. ${ }^{2}$ Laboratory of Clinical Pharmacy and Adverse Drug Reaction, West China Hospital, Sichuan University. ${ }^{3}$ Tianjin Key Laboratory of Metabolic Diseases and Department of Physiology, Tianjin Medical University, Tianjin, China. ${ }^{4}$ Molecular Medicine Research Center, West China Hospital of Sichuan University, Chengdu, China, 610041.

\#, these authors contributed equally to this paper.

\section{*, Corresponding author:}

Jinhan He, Department of Pharmacy, State Key Laboratory of Biotherapy and Cancer Center, West China Hospital of Sichuan University and Collaborative Innovation Center of Biotherapy, Chengdu, Sichuan, China. Email: jinhanhe@scu.edu.cn.

\section{Supplementary Methods \\ Histology analysis}

Liver sections were stained with hematoxylin and eosin (H\&E). Tissue sections were examined in a blinded fashion by a single pathologist and graded for steatosis, inflammation and hepatocyte ballooning as described (1).

\section{References}

1. Kleiner DE, Brunt EM, Van Natta M, Behling C, Contos MJ, Cummings OW, Ferrell LD, et al. Design and validation of a histological scoring system for nonalcoholic fatty liver disease. Hepatology 2005;41:1313-1321. 


\section{Supplementary Figure Legends}

Supplementary Figure 1. LTB $_{4}$ levels were elevated during HSCs activation. Primary HSCs were isolated from wild-type (WT) mice and cultured for 2 days (quiescent HSCs [q-HSCs]) or 7 days (activated HSCs [a-HSCs]). Supernatants were collected from q-HSCs or a-HSCs for metabolomic analysis. Data are mean $\pm \mathrm{SEM}, \mathrm{n}=5,{ }^{*} p<0.05,{ }^{* *} p<0.01$.

Supplementary Figure 2. LTB $_{4}$ and $\mathrm{LTC}_{4}$ levels did affect the ERK pathway but not SMAD and AKT pathway.(A) Western blot analysis of protein levels of p-SMAD, SMAD, p-AKT, AKT, p-ERK1/2, ERK1/2, and $\beta$-tubulin in HSC after treatment with $\mathrm{LTB}_{4}, \mathrm{LTC}_{4}$ or TGF- $\beta 1$ for 30 min. (B) Western blot analysis of the expression of p-ERK1/2 and ERK1/2 with $\mathrm{LTB}_{4}$ or $\mathrm{LTC}_{4}$ treatment. HSC were pre-treated with PD98059 for $1 \mathrm{hr}$ then $\mathrm{LTB}_{4}$ or $\mathrm{LTC}_{4}$. (C) Western blot analysis of protein levels of p-P38, p-JNK, and $\beta$-tubulin after treatment with $\mathrm{LTB}_{4}$, $\mathrm{LTC}_{4}$ or TGF- $\beta 1$ for $30 \mathrm{~min}$.

\section{Supplementary Figure 3. 5-LO was upregulated in NASH-mice liver.}

(A) mRNA level of 5-LO in WT mice fed an MCS or MCD diet for 6 weeks. (B) Protein levels of 5-LO, Flap, Collal and $\alpha$-SMA in livers of WT mice fed an MCS and MCD diet for 6 weeks. (C) Western blot quantification of 5-LO expression in Supplementary Figure 3B. Data are mean $\pm \mathrm{SEM}, \mathrm{n}=5,{ }^{* *} p<0.01$.

\section{Supplementary Figure 4. The effect of supernatant from 5- $\mathrm{LO}^{-/}$and WT a-HSC activation} on HSC activation.

Primary HSC were isolated from WT mice and 5- $\mathrm{LO}^{-/}$mice and cultured for 7 days. The

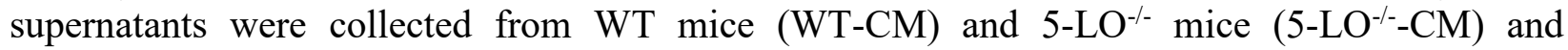
incubated with primary HSC from WT mice and $5-\mathrm{LO}^{-/}$mice for 7 days. (A-B) The mRNA and protein levels of fibrotic markers were detected. Data are mean $\pm \mathrm{SEM}, \mathrm{n}=5,{ }^{*} p<0.05,{ }^{* *} p<$ 0.01 .

\section{Supplementary Figure 5. Genetic ablation of 5-LO ameliorated liver injury and} inflammation with $\mathrm{CCl}_{4}$ treatment.

WT and 5- $\mathrm{LO}^{-/}$mice were injected with olive oil or $\mathrm{CCl}_{4}$ for 8 weeks. Serum level of ALT and AST levels (A) and inflammatory cell infiltration (B) with $\mathrm{CCl}_{4}$ treatment. (C) Liver sections were stained with F4/80 and $\alpha$-SMA.(D) qRT-PCR analysis of mRNA levels of TNF- $\alpha$, IL- $1 \beta$, Mcp-1, and Cd68. Data are mean \pm SEM, $\mathrm{n}=5,{ }^{*} p<0.05,{ }^{* *} p<0.01$.

\section{Supplementary Figure 6. Genetic ablation of 5-LO ameliorated MCD diet-induced liver} fibrosis. WT and 5-LO $\%$ mice were fed an MCS or MCD diet for 6 weeks. Fibrosis stage was assessed after picrosirius red staining (A-B) for collagen according to the Ishak criteria (C). (D) The detection of hepatic hydroxyproline levels. (E-F) qRT-PCR analysis of mRNA levels of fibrotic genes in livers of 4 groups. $(\mathrm{G})$ Western blot analysis of protein levels of $\alpha$-SMA and Colla1. (H) Western blot quantification of $\alpha$-SMA and Colla1 expression in Supplementary Figure 5F. Data are mean \pm SEM, $\mathrm{n}=7,{ }^{*} p<0.05,{ }^{* *} p<0.01$.

\section{Supplementary Figure 7. Genetic ablation of 5-LO ameliorated liver injury and} inflammation with MCD diet. WT and 5-LO/- mice were fed an MCS or MCD diet for 6 weeks. qRT-PCR analysis of the effect of genetic ablation of 5-LO on MCD diet-induced liver injury (A) 
and inflammatory cell infiltration (B). (C) NAFLD Activity Score for degree of steatosis, lobular inflammation, and hepatocyte ballooning. (D) qRT-PCR analysis of mRNA levels of proinflammatory genes in livers of $\mathrm{WT}$ and $5-\mathrm{LO}^{-/}$mice. Data are mean $\pm \mathrm{SEM}, \mathrm{n}=7, * p<0.05$, $* * p<0.01$.

Supplementary Figure 8. The characterization of liposomes. (A) Schematic illustration of RGD-Lip/zileuton. (B) The morphology of liposomes.

Supplementary Figure 9. RGD-Lip/zileuton inhibits secretion of $\mathrm{LTB}_{4}$ in a-HSCs. Primary HSCs were isolated from WT mice and cultured for 7 days to activation. Cells were pretreated with RGD-Lip or RGD-Lip/zileuton $(10 \mu \mathrm{M})$ for $48 \mathrm{hr}$. The supernatants were collected for $\mathrm{LTB}_{4}$ detection. Data are mean \pm SEM, $\mathrm{n}=5, * p<0.05$.

Supplementary Figure 10. Targeted delivery of zileuton to $\mathrm{HSCs}$ in $\mathrm{CCl}_{4}$-induced fibrotic model. (A) The regimen of RGD-Lip/zileuton treatment. $\mathrm{CCl}_{4}$ injection lasted for 8 weeks. During the last 4 weeks, mice were administered RGD-Lip or RGD-Lip/zileuton $(10 \mathrm{mg} / \mathrm{kg})$ every 3 days by tail vein injection. (B) The concentration of zileuton in HSCs, hepatocytes (MPH), Kupffer cells (KC), liver sinusoidal endothelial cells (LSEC) and biliary duct epithelial cells (BEC). Data are mean \pm SEM, $\mathrm{n}=7, * * p<0.01$.

Supplementary Figure 11. Targeted delivery of zileuton to HSC ameliorated liver injury and inflammation with $\mathbf{C C l}_{\mathbf{4}}$ administration. WT mice were treated as in Supplementary Figure 9A. (A) Serum was collected for ALT and AST detection. (B) Liver sections were isolated for H\&E staining. (C) qRT-PCR analysis of mRNA levels of proinflammatory genes in liver. (D) Liver sections were stained with F4/80 and $\alpha$-SMA.Data are mean \pm SEM, $\mathrm{n}=7,{ }^{*} p<$ $0.05, * * p<0.01$.

Supplementary Figure 12. Targeted delivery of zileuton to HSC in MCD diet model. The regimen of RGD-Lip/zileuton treatment. WT mice were fed an MCS or MCD diet for 6 weeks. During the last 4 weeks, mice were treated with RGD-Lip or RGD-Lip/zileuton $(10 \mathrm{mg} / \mathrm{kg})$ every 3 days by tail vein injection.

Supplementary Figure 13. Targeted delivery of zileuton to HSC was efficient against MCD diet-induced liver fibrosis. WT mice were treated as in Supplementary Figure 11. Fibrosis stage was assessed after picrosirius red staining (A-B) for collagen according to the Ishak criteria (C). (D) Liver hydroxyproline was detected to reflect the degree of collagen deposition in the liver. (E-F) qRT-PCR analysis of mRNA levels of fibrotic genes in livers of 4 groups. (G-H) Western blot analysis of protein levels of $\alpha$-SMA and Colla1. Data are mean $\pm \mathrm{SEM}, \mathrm{n}=7,{ }^{*} p<0.05$, $* * p<0.01$.

Supplementary Figure 14. Targeted delivery of zileuton to HSCs ameliorated liver injury and inflammation with MCD diet. WT mice were treated as in Supplementary Figure 11. Effect of inhibition of 5-LO on serum ALT and AST levels (A) and H\&E staining (B) of MCD diet-induced liver injury. (C) Inhibition of 5-LO in HSCs decreased hepatic steatosis, lobular inflammation, ballooning and NAFLD score. (D) qRT-PCR analysis of mRNA levels of proinflammatory genes in livers. Data are mean $\pm \mathrm{SEM}, \mathrm{n}=7,{ }^{*} p<0.05,{ }^{* *} p<0.01$. 
Supplementary Figure 15. Human tissue for $\mathrm{H} \& \mathrm{E}$ and picrosirius red staining.

Livers were collected from normal individuals or patients with NASH and fibrosis for H\&E (A) and picrosirius red staining (B). The Sirus red positive area were detected by Image J. Data are mean \pm SEM, $\mathrm{n}=3-5, * * p<0.01$. 


\section{Supplementary Tables}

Supplementary Table 1: List of antibodies used for western blot analysis and immunostaining.

\begin{tabular}{llll}
\hline Antibodies & Application & Company & CAT. No. \\
\hline Anti-a-SMA & WB, IF & SIGMA & A5228 \\
\hline Anti-a-SMA & IF & HUABIO & ER1003 \\
\hline Anti-p-tubulin & WB & Zen BioScience & 200608 \\
\hline Anti-Col1a1 & WB & Bosterbio & PB0981 \\
\hline Anti-5-Lipoxygenase & WB, IF & CST & 3289 \\
\hline Anti-5-Lipoxygenase & IF & SANTA & sc-136195 \\
\hline Anti-p-Erk1/2 & WB & SANTA & sc-7383 \\
\hline Anti-t-Erk1/2 & WB & SANTA & sc-93 \\
\hline Anti-p-Akt & WB & SANTA & sc-7985 \\
\hline Anti-t-Akt & WB & Bosterbio & BM4400 \\
\hline Anti-p-Smad2/3 & WB & CST & 58285 \\
\hline Anti-t-Smad2/3 & WB & CST & \\
\hline
\end{tabular}


Supplementary Table 2: List of specific primers used for RT-PCR

\begin{tabular}{|c|c|c|}
\hline Genes & Sense (5'- 3') & anti-sense (5'- $\left.3^{\prime}\right)$ \\
\hline ma-SMA & CAGGGAGTAATGGTTGGAAT & TCTCAAACATAATCTGGGTCA \\
\hline mCol1a1 & CATGAGCCGAAGCTAACCC & TGTGGCAGATACAGATCAAGC \\
\hline mCol3a1 & TGCTCCTGTGCTTCCTGATG & GACCTGGTTGTCCTGGAAGG \\
\hline mCol5a1 & CCTGCCTGACTCATCTGTGC & AGGCTGTGGTGTGGCTAGGT \\
\hline mTGF- $\beta 1$ & ATGGTGGACCGCAACAAC & AGCCACTCAGGCGTATCAG \\
\hline mTimp-1 & GCAACTCGGACCTGGTCATAA & CGGCCCGTGATGAGAAACT \\
\hline mTimp-2 & TCAGAGCCAAAGCAGTGAGC & GCCGTGTAGATAAACTCGATGTC \\
\hline mPai-1 & TTCAGCCCTTGCTTGCCTC & ACACTTTTACTCCGAAGTCGGT \\
\hline mAlox5 & CACGGGGACTACATCGAGTT & GTGCTGCTTGAGGATGTGAA \\
\hline mMcp1 & GCTGGAGAGCTACAAGAGGATC & GTCAACTTCACATTCAAAGGTGC \\
\hline mTnf-a & GGCGGTGCCTATGTCTCA & AGGGTCTGGGCCATAGAA \\
\hline mIL-1及 & GCAACTGTTCCTGAACTCAACT & ATCTTTTGGGGTCCGTCAACT \\
\hline mCD68 & CAAGGGGGCTCTTGGGAACTA & GCTCTGATGTAGGTCCTGTTTG \\
\hline m18S & TTGACTCAACACGGGAAACC & AGACAAATCGCTCCACCAAC \\
\hline
\end{tabular}


Supplementary Table 3: Particle size and zeta potential of liposomes.

\begin{tabular}{lccc}
\hline Type & $\begin{array}{c}\text { Particle size } \\
(\mathbf{n m})\end{array}$ & Zeta Potential (mV) & Encapsulation efficiency \\
\hline RGD-Lip & $77.33 \pm 1.561$ & -17.5 & $/$ \\
\hline RGD-Lip/zileuton & $72.37 \pm 2.248$ & -14.8 & $70.42 \pm 0.722$ \\
\hline
\end{tabular}

Data are mean \pm SD 
Supplementary Figure 1

A
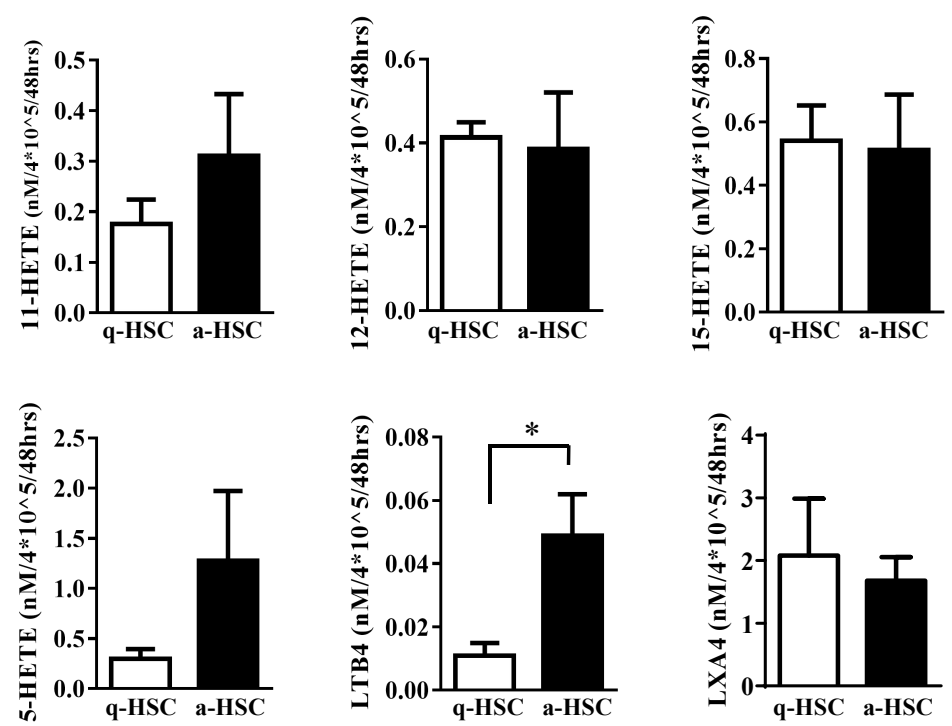


\section{Supplementary Figure 2}

A

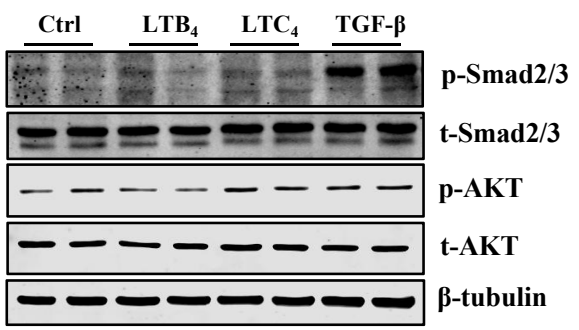

$\underline{\text { Ctrl }} \quad$ LTB $_{4} \quad$ LTC $_{4} \quad \underline{\text { TGF- } \beta}$

$=E=\bar{E}=-E$ p-Ekk1/2

Erk1/2

- $-\infty-\infty-\infty$-tubulin

B

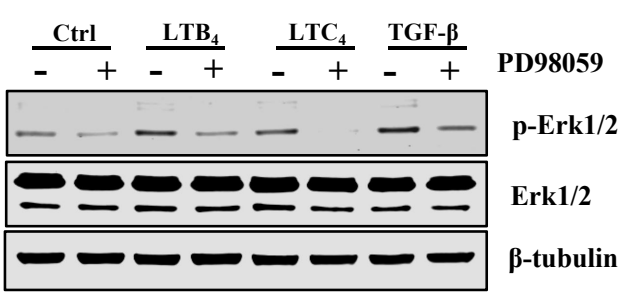

C

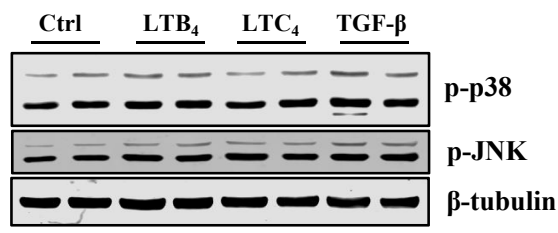


Supplementary Figure 3

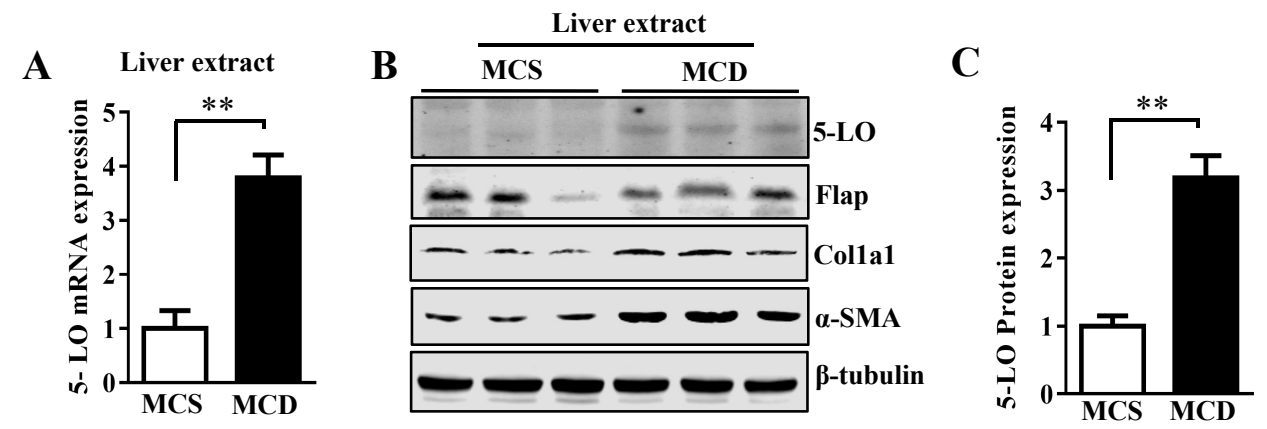


Supplementary Figure 4

A

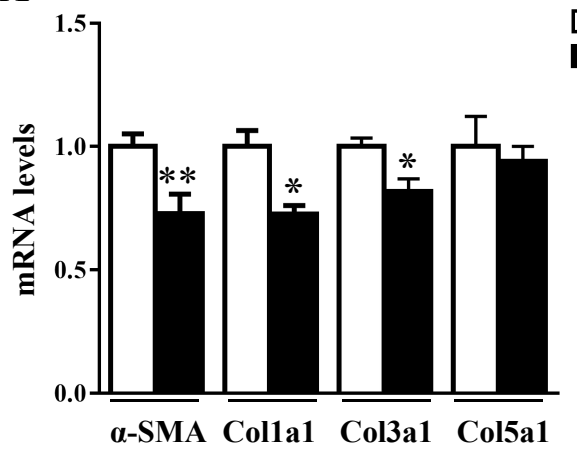

B

WT-CM

5-LO $\mathrm{O}^{-/-}-\mathrm{CM}$

HSC extract

WT-CM 5-LO---CM

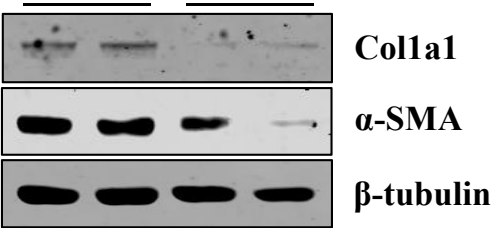




\section{Supplementary Figure 5}

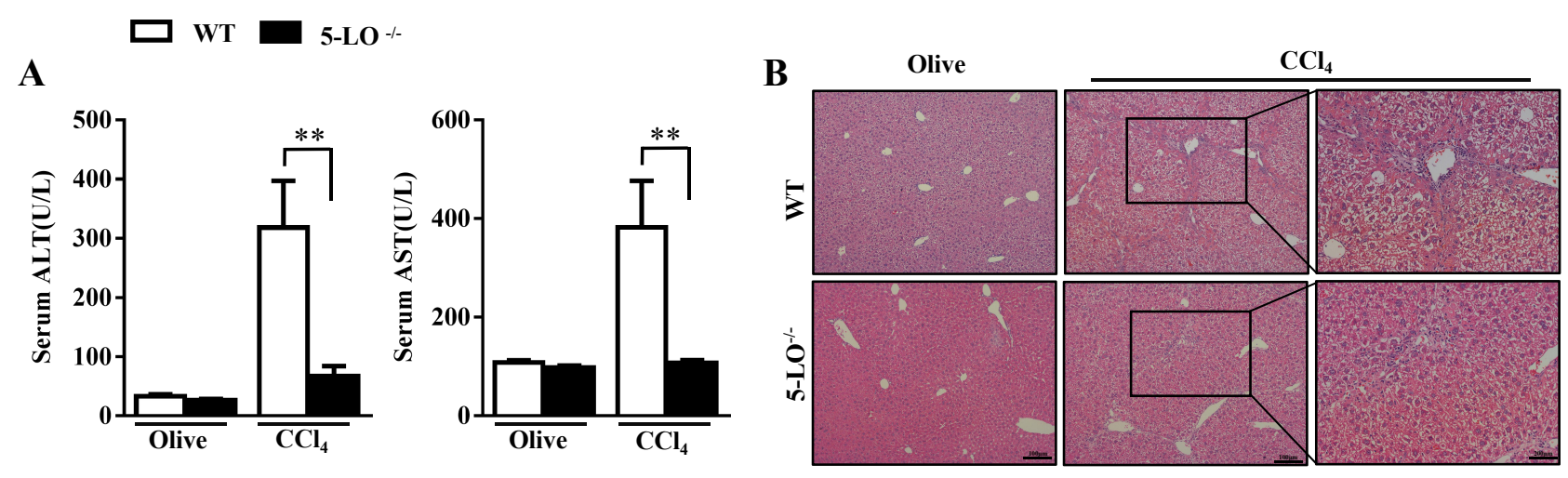

C

F4/80

$\alpha$-SMA

DAPI Merge

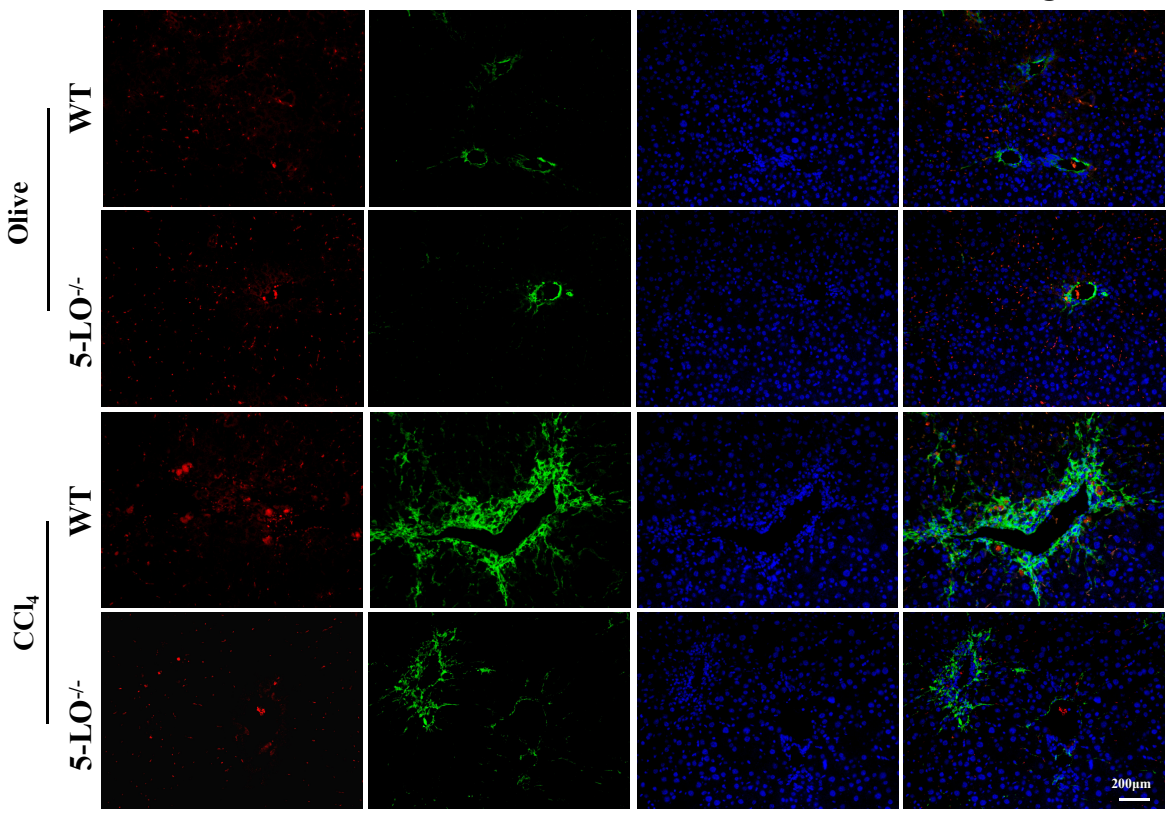

D
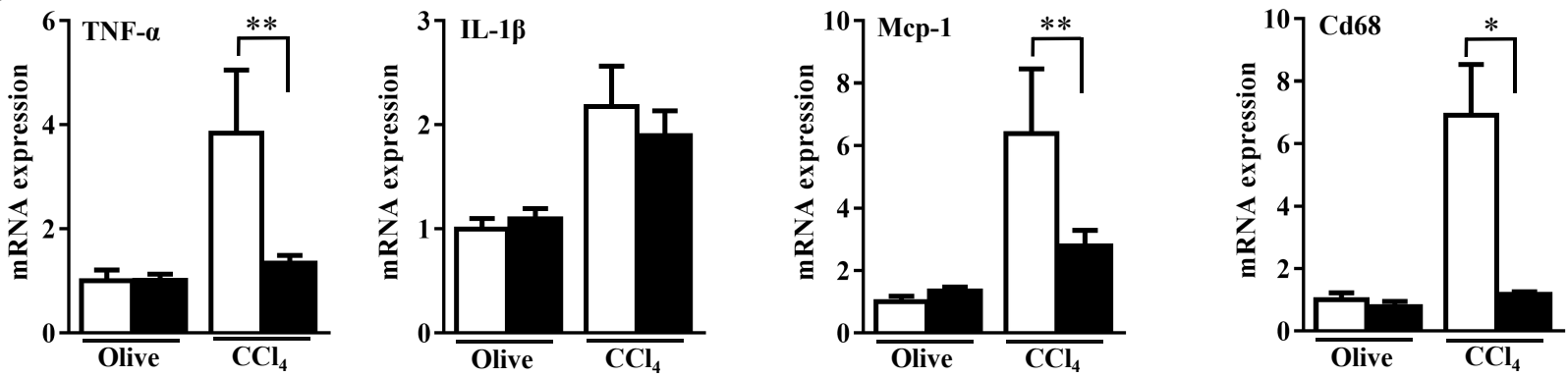
Supplementary Figure 6

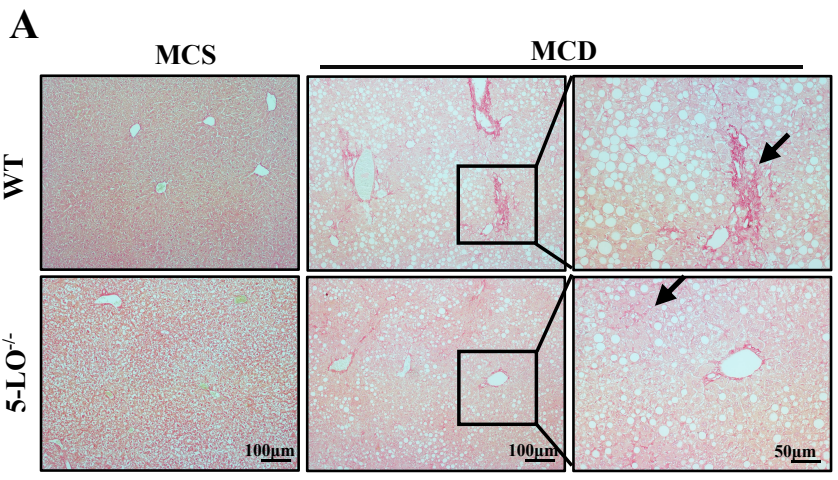

B

C

D
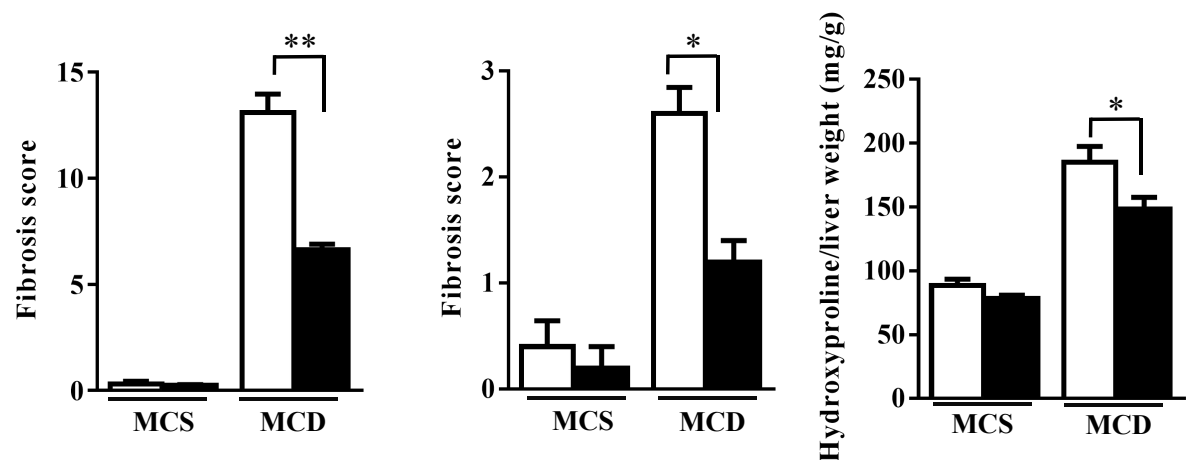

$\square$ WT
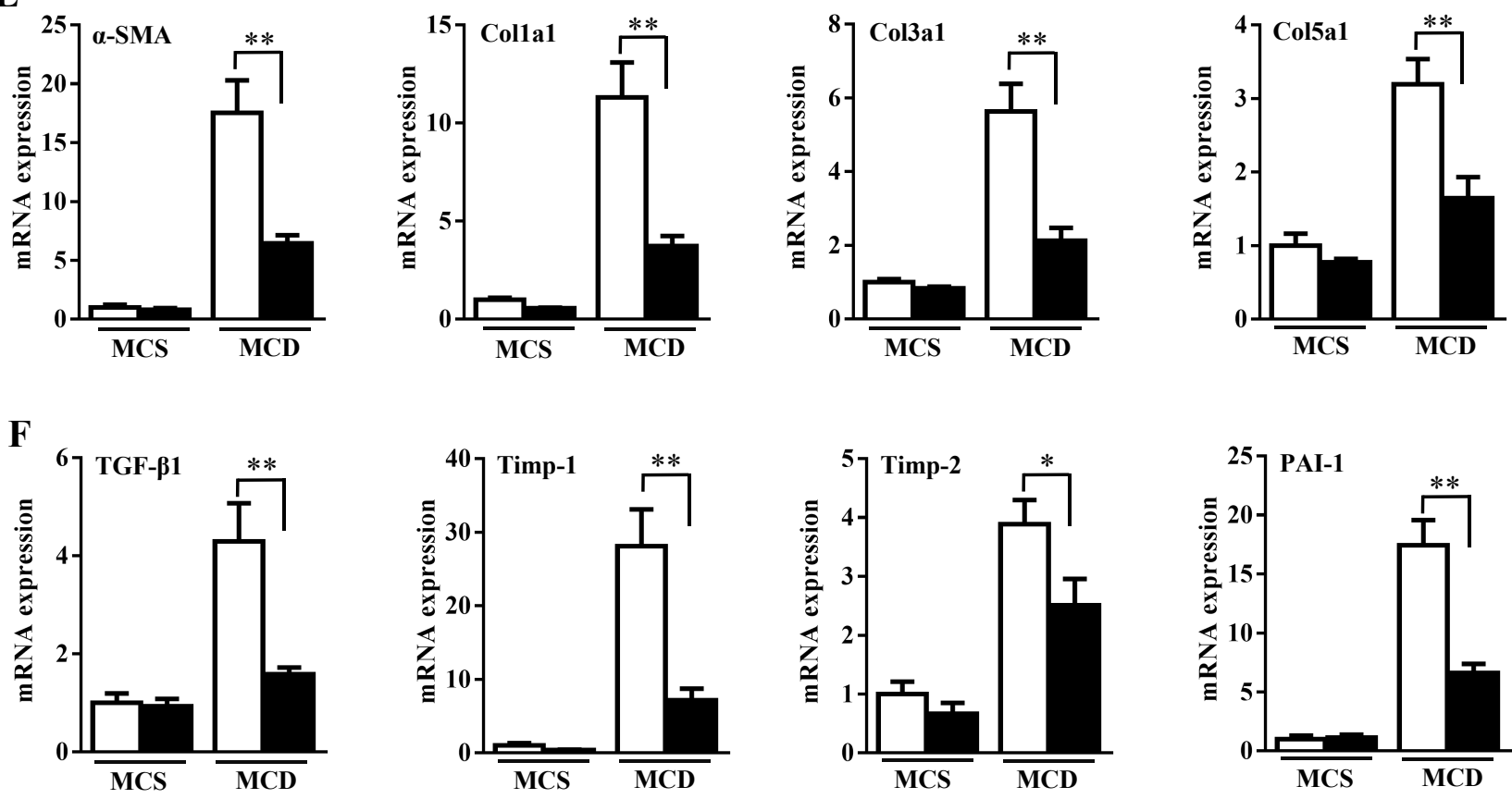

G

H
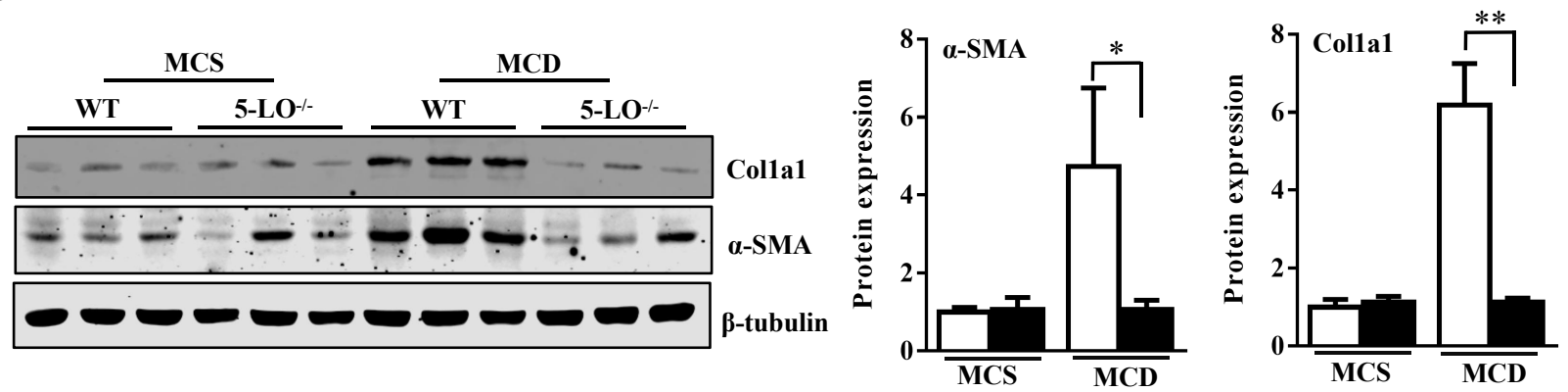
Supplementary Figure 7

A $\square$ WT $\square$ 5-LO

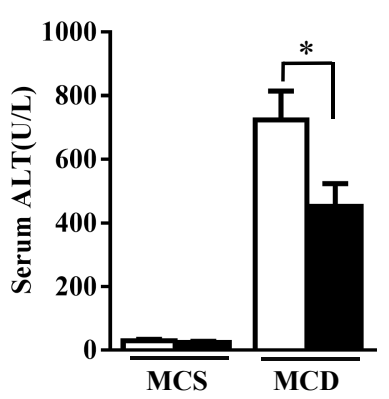

C

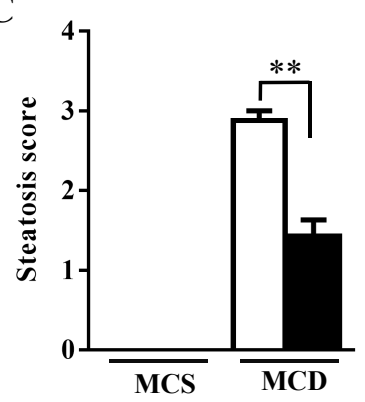

D

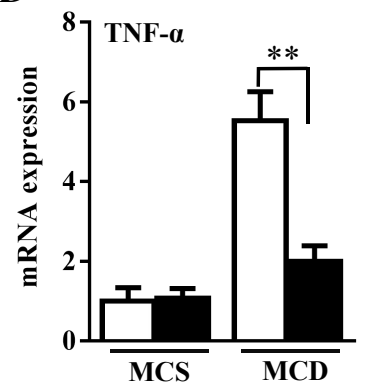

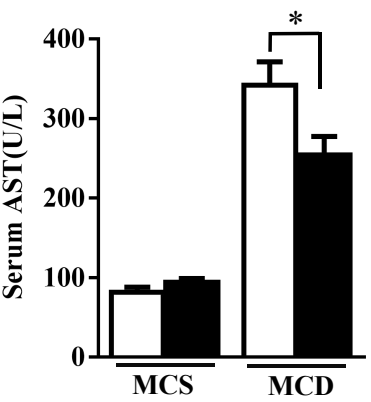

B
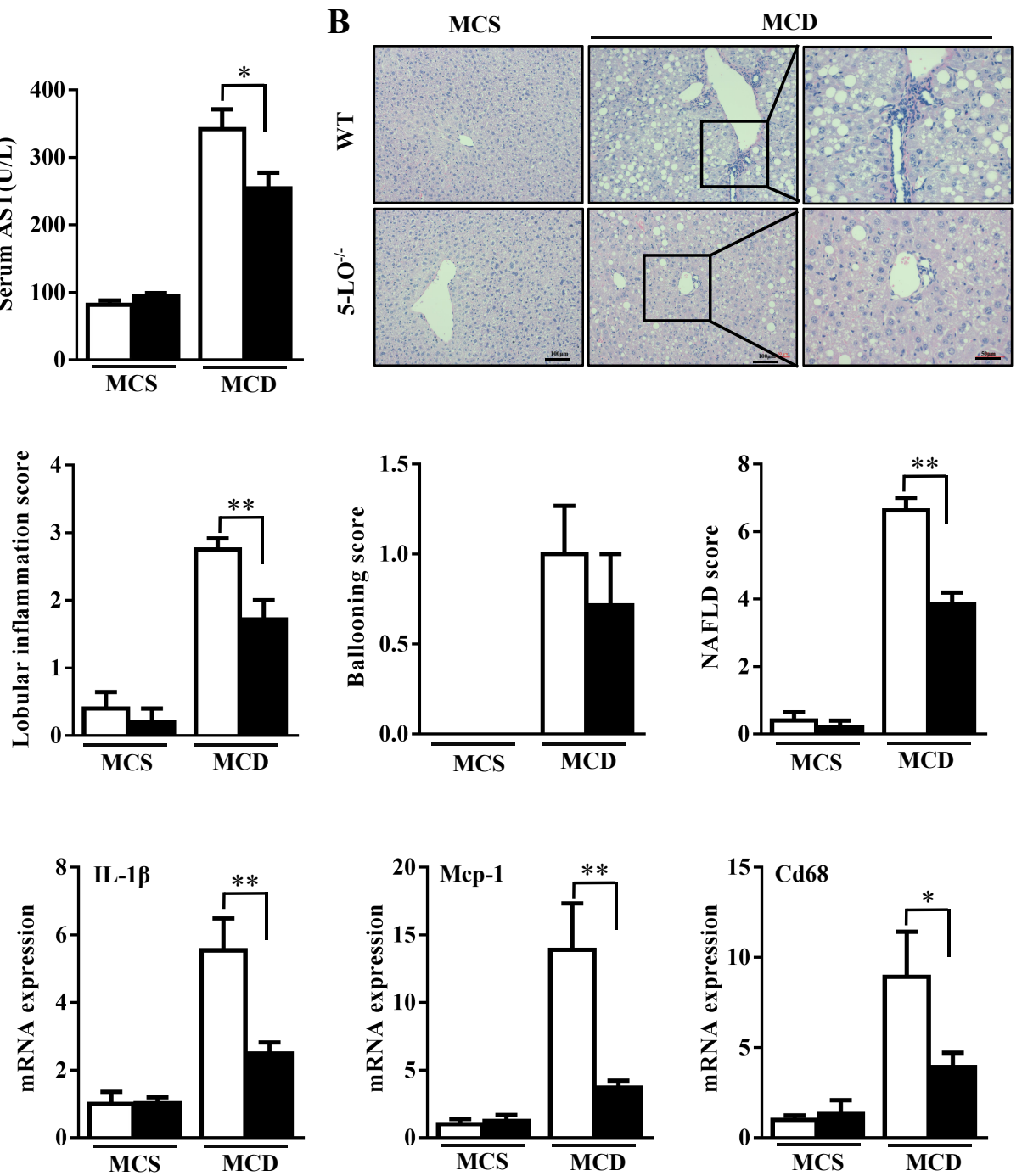


\section{Supplementary Figure 8}

A

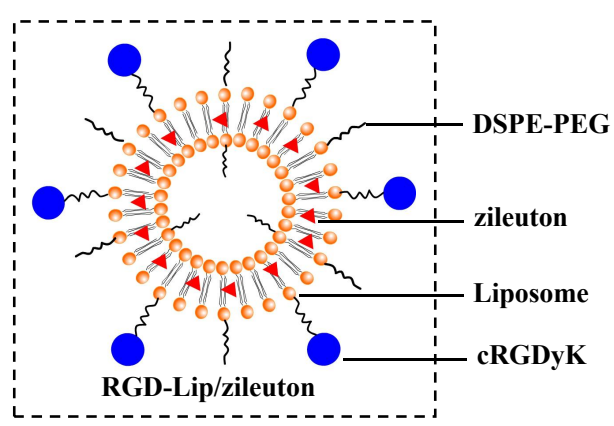

B

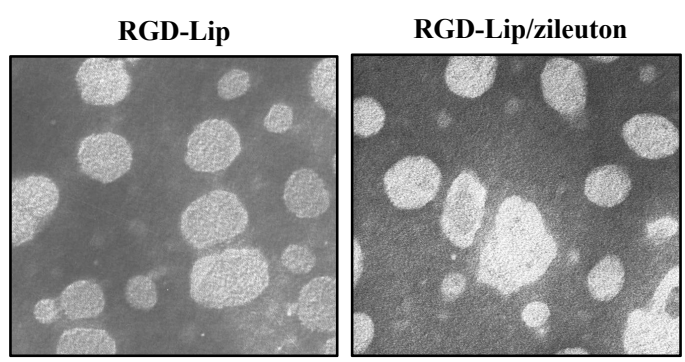


Supplementary Figure 9

$\square$ RGD-Lip

RGD-Lip-Zileuton

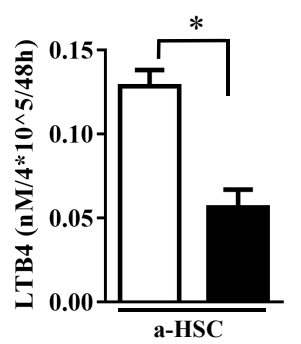




\section{Supplementary Figure 10}

A

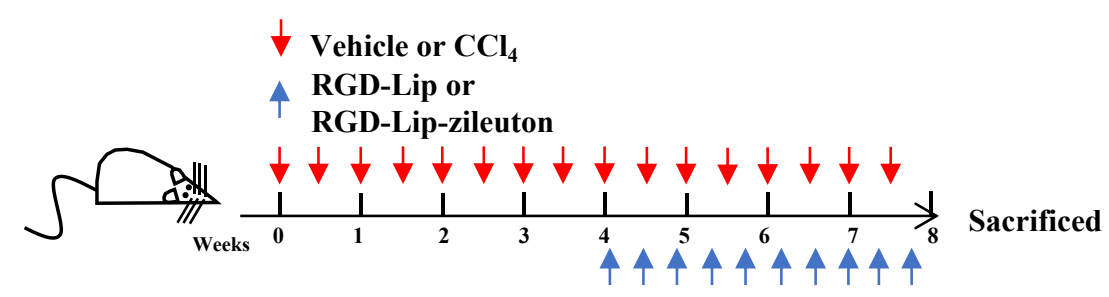

B

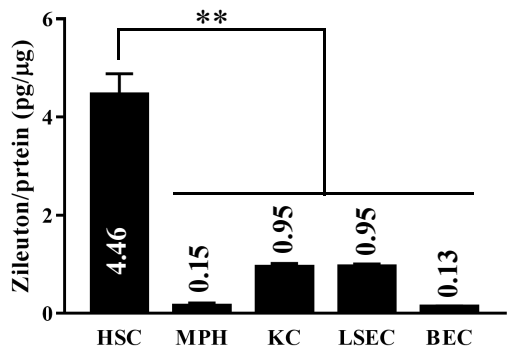




\section{Supplementary Figure 11}
$\square$ RGD-Lip

A
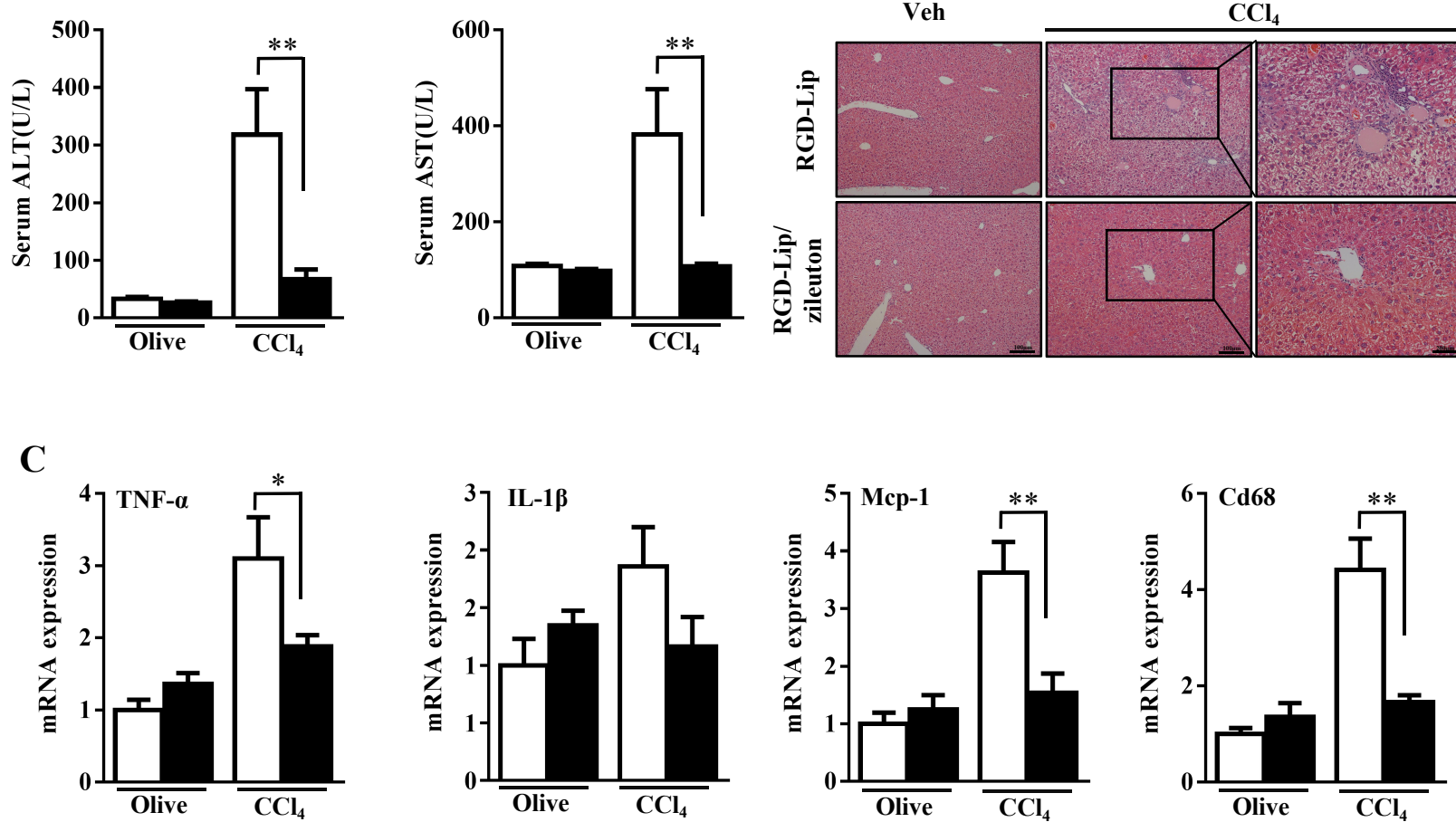

D

F4/80

o-SMA

DAPI

Merge

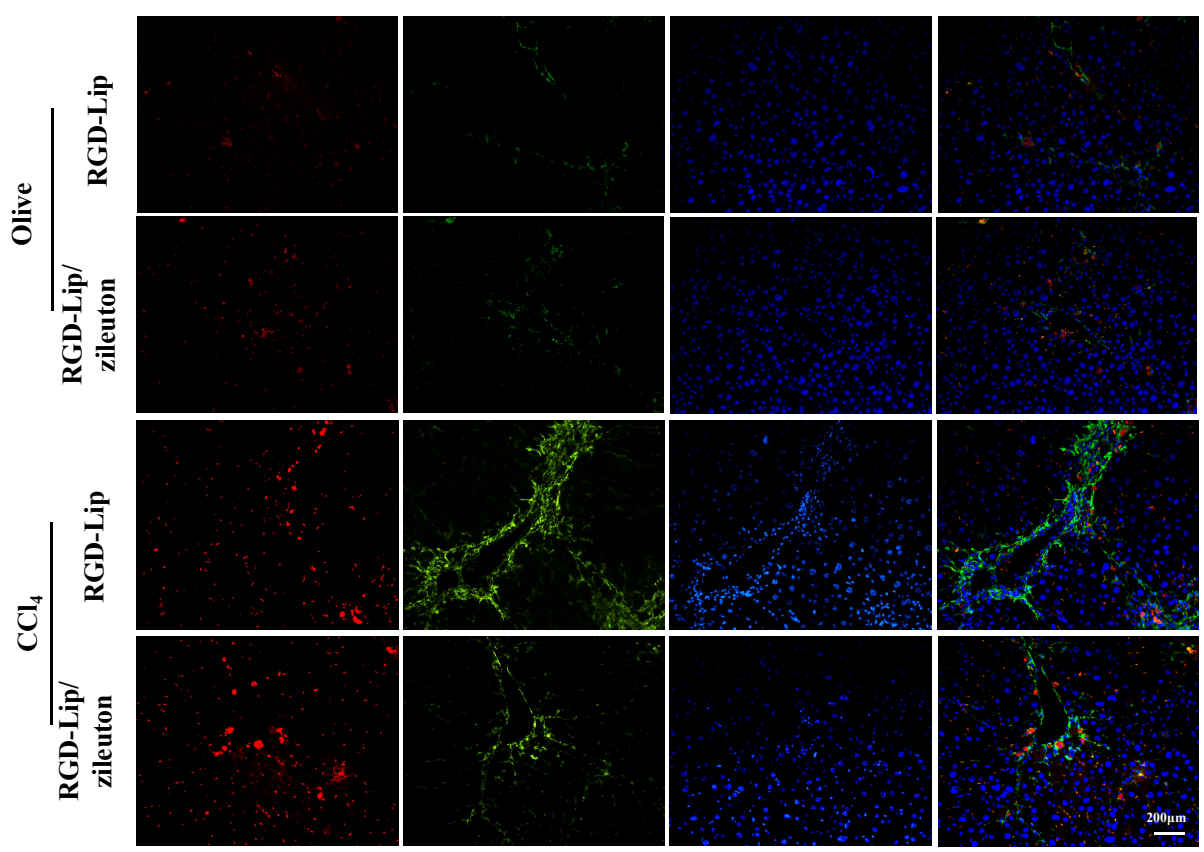


Supplementary Figure 12

$\uparrow$ RGD-Lip or RGD-Lip/Zileuton

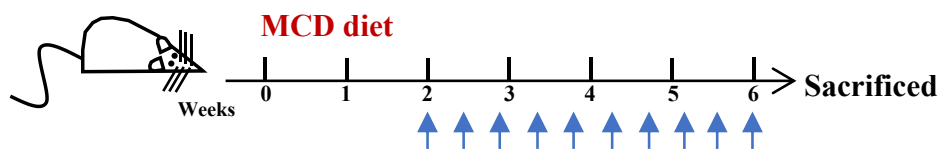


Supplementary Figure 13
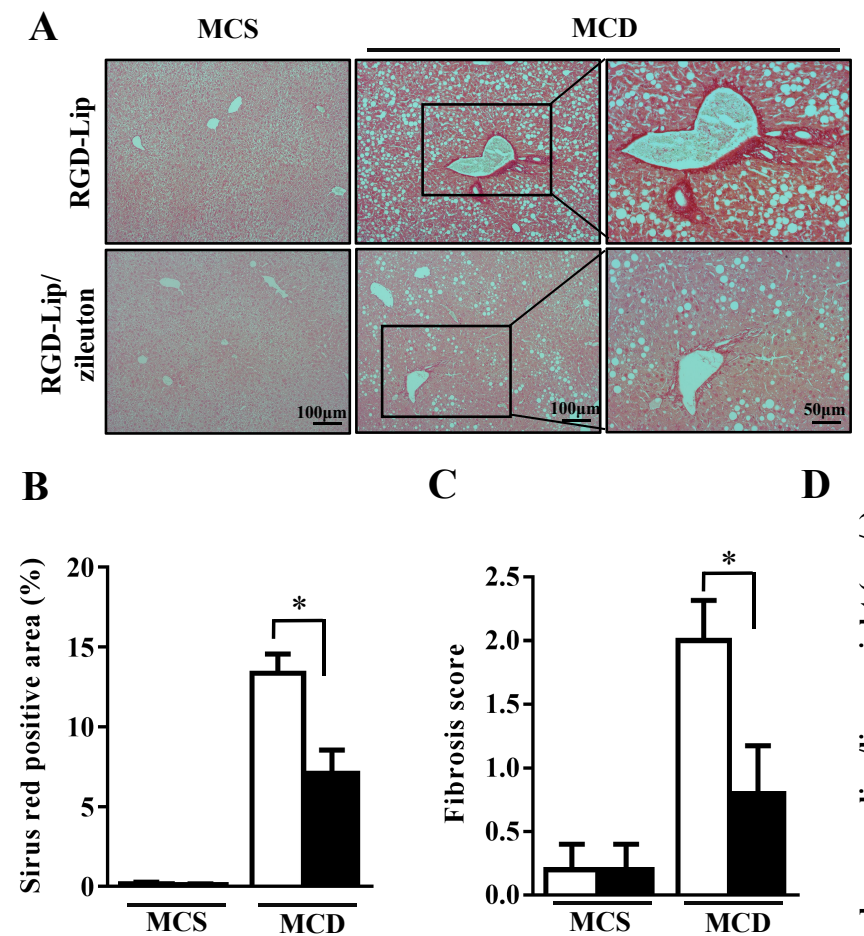

C

D

$\square$ RGD-Lip

E
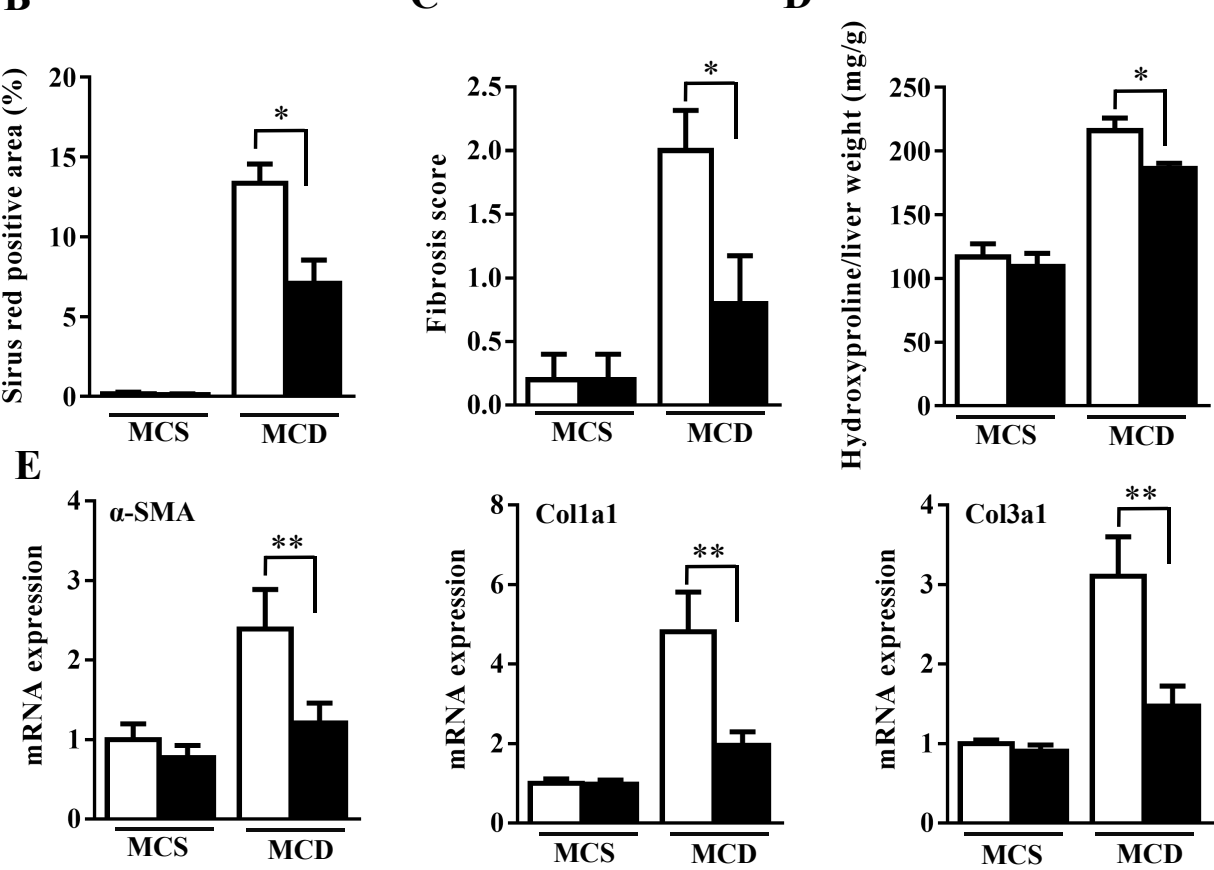

RGD-Lip/zileuton

F
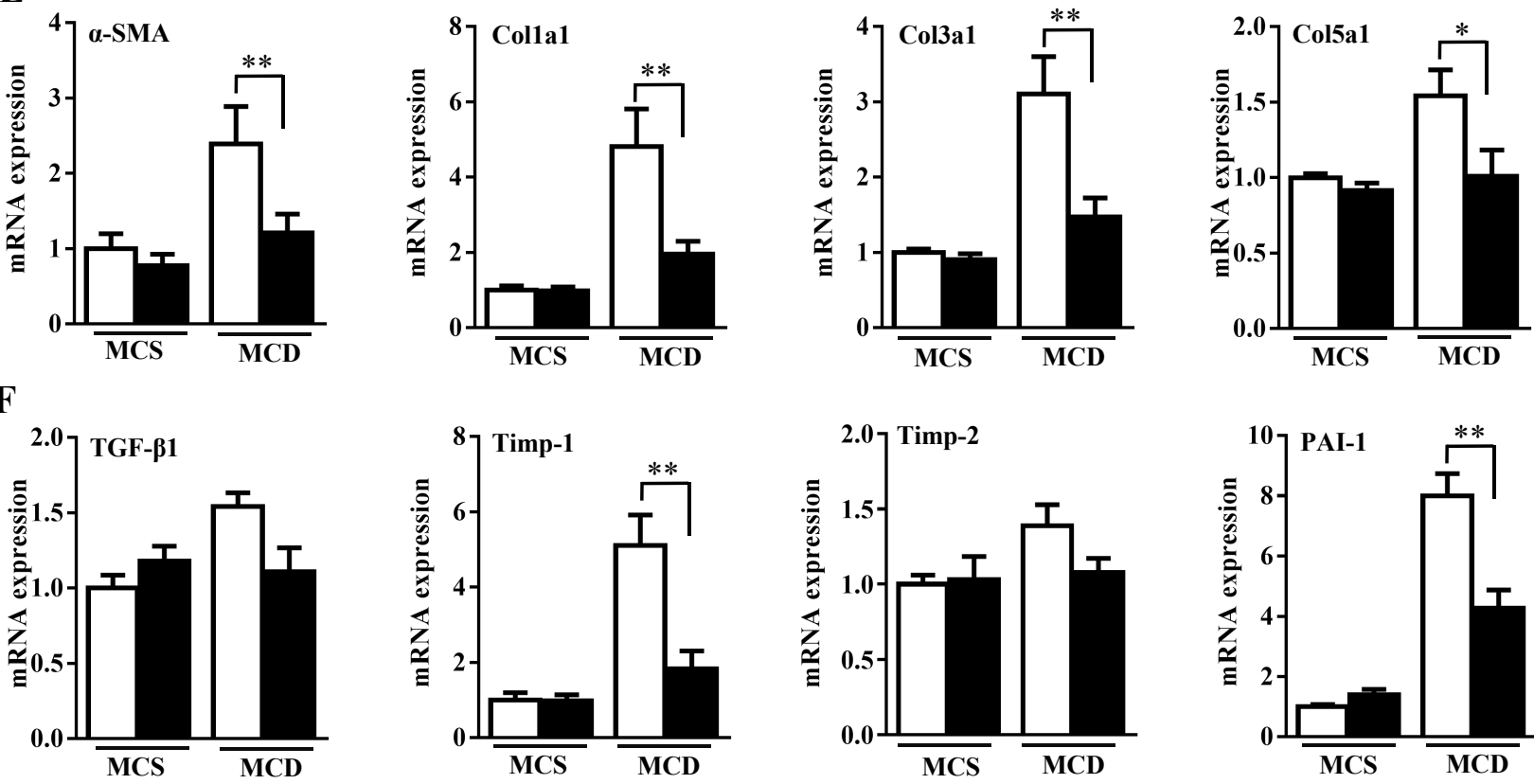

G
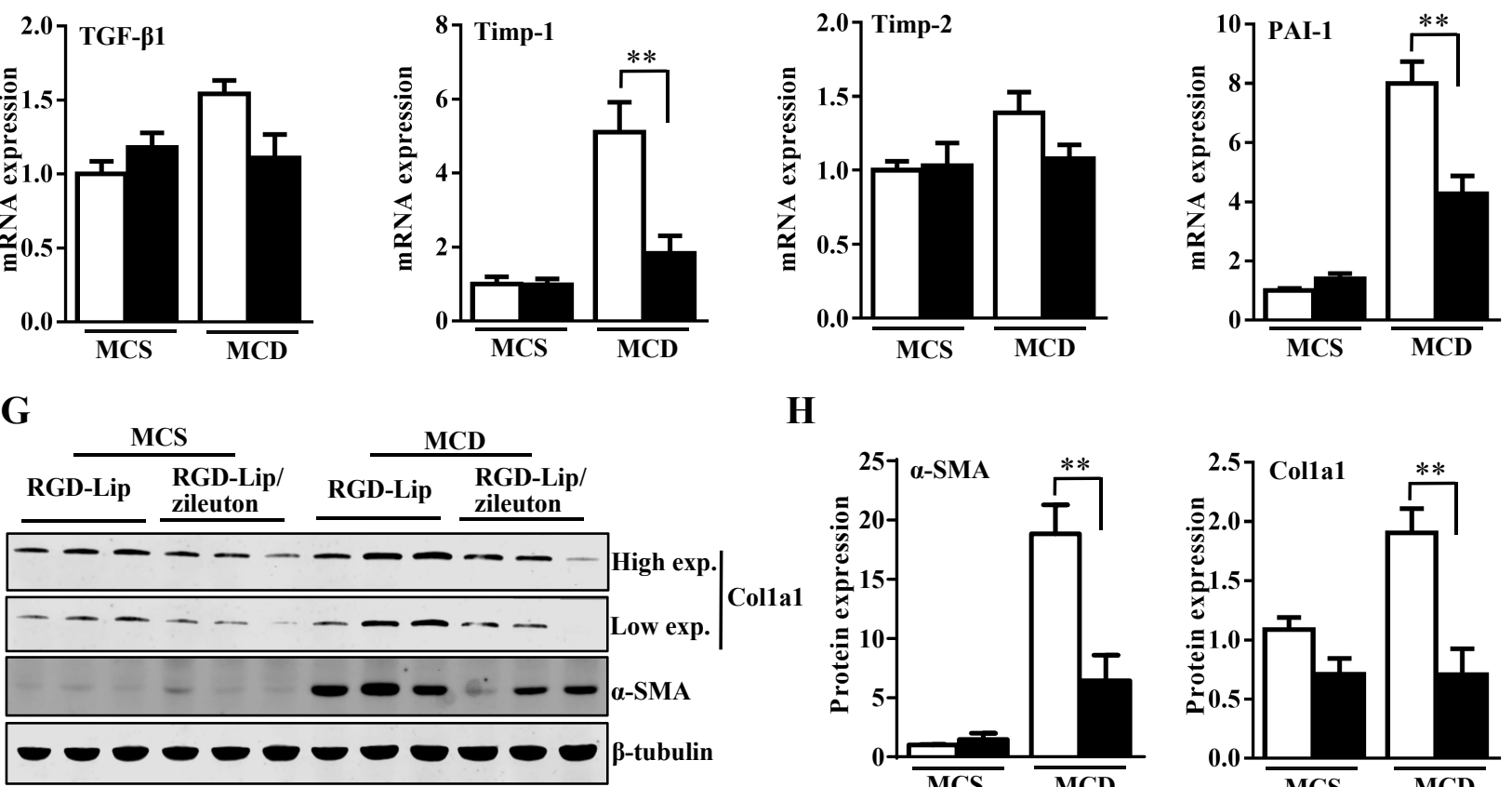

H
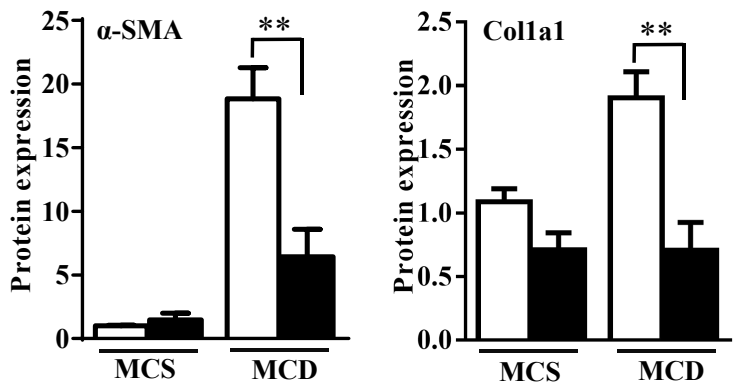
Supplementary Figure 14

A $\square$ RGD-Lip $\square$ RGD-Lip/zileuton

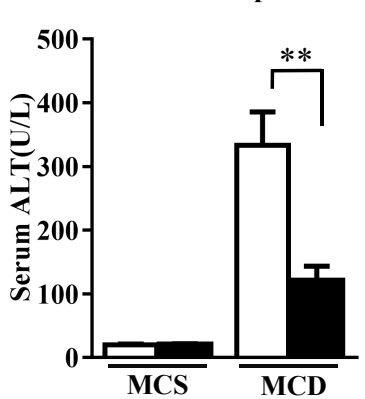

C

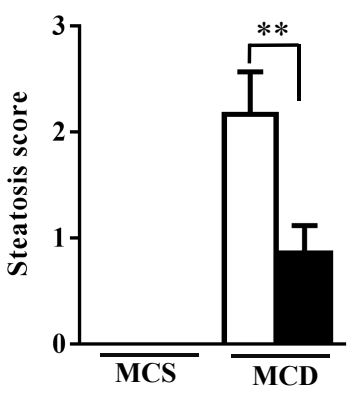

D

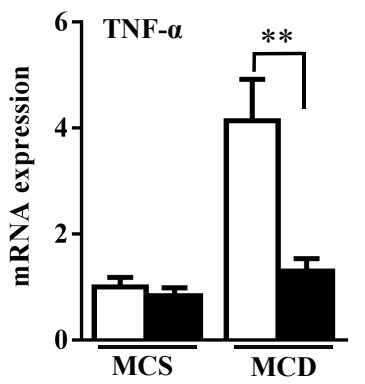

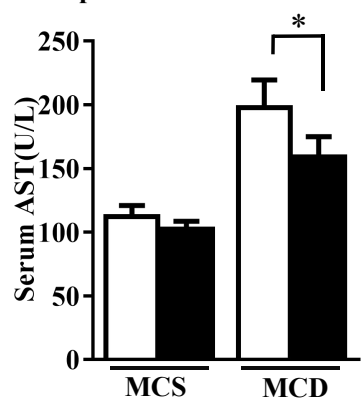
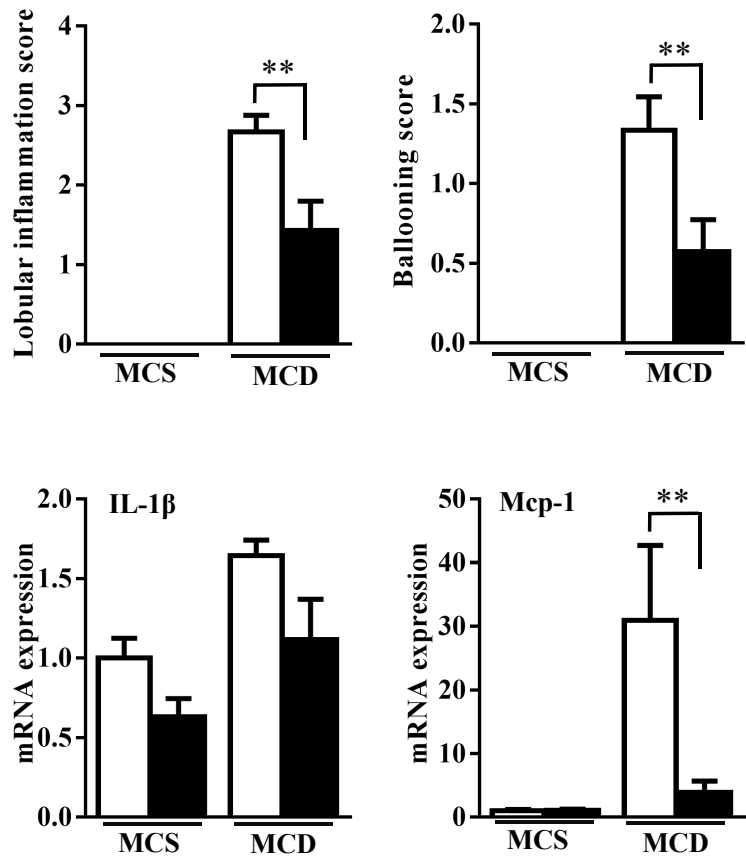
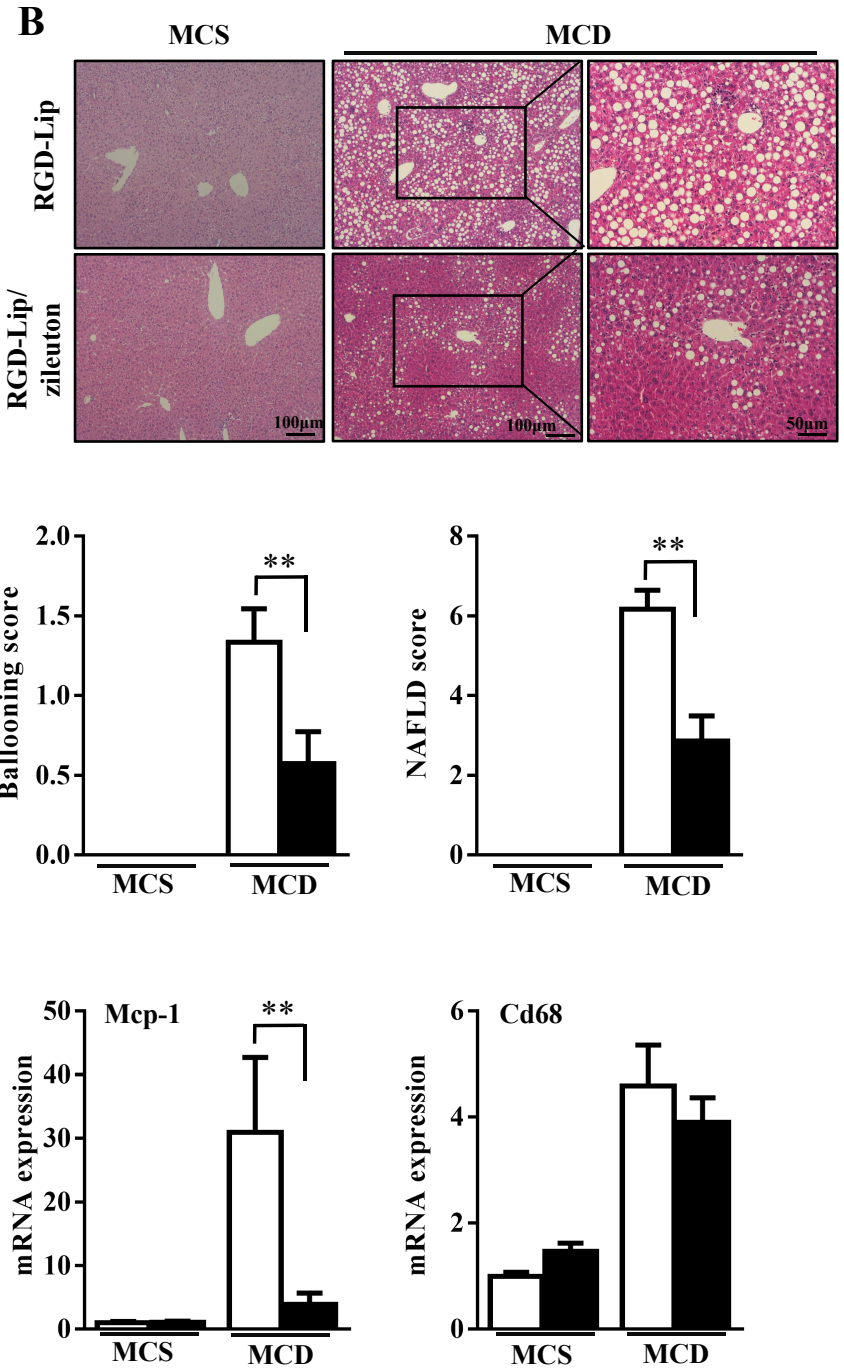
Supplementary Figure 15

A

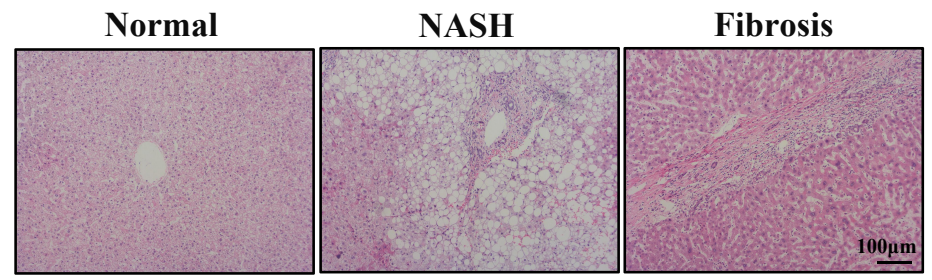

B

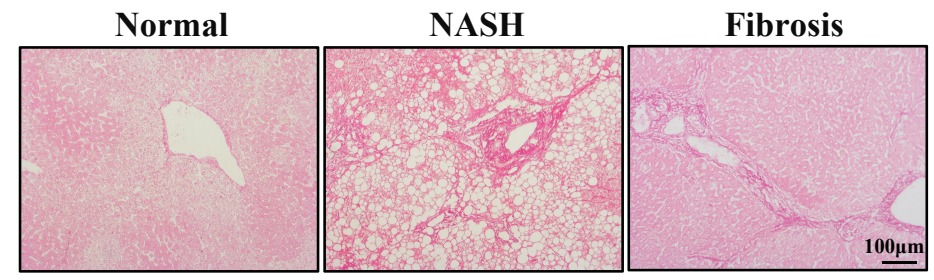

C

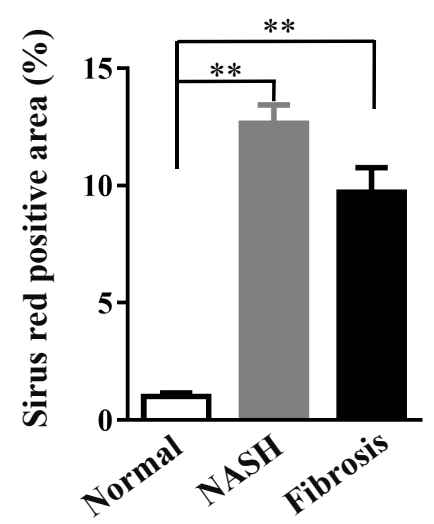

